\title{
S100A4 influences cancer stem cell-like properties of MGC803 gastric cancer cells by regulating GDF 15 expression
}

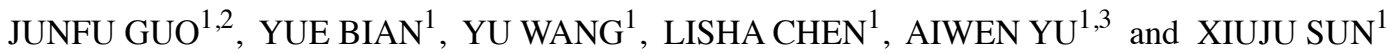 \\ ${ }^{1}$ Department of Medical Genetics, China Medical University, Shenyang, Liaoning $110122 ;{ }^{2}$ Teaching and Experiment Center, \\ Liaoning University of Traditional Chinese Medicine, Shenyang, Liaoning 110847; ${ }^{3}$ Department of Rehabilitation, \\ The First Affiliated Hospital of China Medical University, Shenyang, Liaoning 110001, P.R. China
}

Received March 7, 2016; Accepted April 19, 2016

DOI: $10.3892 /$ ijo.2016.3556

\begin{abstract}
Many studies have revealed that S100A4 is involved in cancer progression by affecting a variety of biological functions. Our previous study showed that S100A4 influences many biological properties of gastric cancer cells; however, the underlying mechanisms are far from clear. In this study, we used cDNA microarray analysis to investigate the global alterations in gene expression in MGC803 gastric cancer cells after siRNA-mediated S100A4 inhibition. Among the total genes investigated, 179 differentially expressed genes (38 upregulated and 141 downregulated) were detected in S100A4-siRNA transfected MGC803 cells compared with NC-siRNA transfected cells. We focused on the GDF15 gene, which was significantly downregulated after S100A4 inhibition. ChIP studies showed that the S100A4 protein binds to the GDF15 promoter, implicating S100A4 in GDF15 regulation at the transcriptional level. GDF15 overexpression promoted CSC-like properties of MGC803 cells, such as spheroid and soft-agar colony forming abilities. S100A4 inhibition suppressed the CSC-like properties of the cells, whereas, GV141-GDF15 vector transfection reversed these effects. Our results suggest that $S 100 A 4$ influences the CSC-like properties of MGC803 gastric cancer cells by regulating GDF15 expression.
\end{abstract}

\section{Introduction}

S100A4 (also known as calvasculin), belongs to the S100 family of $\mathrm{Ca}^{2+}$-binding proteins. The human $5100 A 4$ gene is located in chromosome 1q21. The S100A4 protein occurs as noncovalently bound homodimers with the ability to interact with an array of target proteins in a calcium-dependent manner (1).

Correspondence to: Professor Xiuju Sun, Department of Medical Genetics, China Medical University, 77 Puhe Road, Shenyang North New Area, Shenyang, Liaoning 110122, P.R. China

E-mail: xjsun@mail.cmu.edu.cn

Key words: gastric cancer, S100A4, GDF15, spheroid, soft-agar colony
S100A4 overexpression has been reported in several types of cancer and is associated with invasion and metastasis and poor patient prognosis (2-4). Many studies have confirmed that S100A4 is involved in a variety of biological effects. Knockdown of S100A4 inhibited the invasiveness of esophageal squamous cell carcinoma cells, with elevated $E$-cadherin expression (5). The siRNA-mediated silencing of S100A4 downregulated $M M P-13$ expression and suppressed breast cancer cell migration and angiogenesis (6). Recently, increased expression of P27 and cleaved caspase-3 has been reported in S100A4-deficient pancreatic tumors, while cyclin E expression was found to be decreased. S100A4-deficient tumors have reduced expression of vascular endothelial growth factor (VEGF), suggesting reduced angiogenesis (7). Our group previously showed that S100A4 inhibition mediated by RNA interference (RNAi) led to reduced proliferation and increased apoptosis of BGC823 gastric cancer cells. Intratumoral injection of pS100A4-shRNA suppressed tumor growth in nude mice. We also found that $S 100 A 4$ inhibition decreased expression of both $N F-\kappa B$ p 65 and phosphorylated (Ser32) $-I-\kappa B-\alpha$ in BGC823 cells (8). These studies indicate that $\$ 100 A 4$ exerts its function by affecting downstream gene expression although the mechanisms remain to be fully clarified.

Accumulating evidence suggests that cancer-initiating cells (CIC) or cancer stem cells (CSC) are a rare subpopulation of cells with self-renewal capacity $(9,10)$ and are responsible for cancer initiation, progression, metastasis, relapse, radioresistance and chemoresistance (11-13). S100A4 knockdown in head and neck CICs reduced their stemness properties both in vitro and in vivo (14). Our previous study showed that S100A4 mediated the effects of IL-1 $\beta$ on the CSC-like properties of MGC803 gastric cancer cells (unpublished data); however, the mechanisms underlying this effect are far from clear. In this study, we investigated the hypothesis that $5100 A 4$ affects the CSC-like properties of MGC803 gastric cancer cells via regulation of downstream gene expression.

In this study, cDNA microarray analysis showed differential expression of 179 of the total genes after siRNA-mediated S100A4 knockdown in MGC803 gastric cancer cells. We then focused specifically on the GDF15 gene, which was significantly downregulated after S100A4 inhibition. ChIP assays showed that S100A4 protein binds to the GDF15 promoter, indicating that S100A4 may participate in the transcriptional 
regulation of GDF15. GDF15 overexpression promoted the CSC-like properties of MGC803 cells, such as spheroid and soft-agar colony forming abilities. Finally, rescue experiments indicated that S100A4 influences the CSC-like properties of MGC803 gastric cancer cells by regulating GDF15 expression.

\section{Materials and methods}

Cell culture. The human gastric cancer cell line MGC803 was purchased from the Cell Resource Center, Institute of Basic Medical Sciences (IBMS), Chinese Academy of Medical Sciences and Peking Union Medical College (CAMS/PUMC). Cells were cultured in RPMI-1640 medium (Invitrogen, Carlsbad, CA, USA) supplemented with $10 \%$ fetal bovine serum at $37^{\circ} \mathrm{C}$ in a humidified incubator containing $5 \% \mathrm{CO}_{2}$.

Transfection of S100A4-specific small interfering RNA(siRNA). Duplex siRNA oligos specific for human S100A4 were synthesized by GenePharma (Shanghai, China). The siRNA sequences were as follows: 5'-GCAUCGCCAUGAUGUGUA ATT-3', and 5'-UUACACAUCAUGGCGAUGCTT-3'. Negative control (NC) siRNAs were provided by GenePharma. MGC803 cells were transfected with $20 \mathrm{nM}$ of siRNA using Lipofectamine $^{\mathrm{TM}} 2000$ transfection reagent (Invitrogen) according to the manufacturer's instuctions. The cells transfected with S100A4-siRNA or NC-siRNA were referred to as MGC803/S100A4-siRNA cells or MGC803/NC-siRNA cells, respectively. Cells were harvested at $48 \mathrm{~h}$ after transfection for use in the subsequent associated experiments.

RNA extraction and quantitative reverse transcription polymerase chain reaction ( $q R T-P C R)$. Total cellular RNA was extracted using TRIzol reagent (Invitrogen). Reverse transcription reaction was performed using the First-Strand cDNA synthesis kit (Promega, Madison, WI, USA) with $1 \mu \mathrm{g}$ of RNA in a final volume of $20 \mu \mathrm{l}$. The newly synthesized cDNA was amplified by quantitative PCR and PCR analysis was carried out using SYBR Premix Ex TaqII (Takara Biotechnology, Tokyo, Japan). Reactions were processed and analyzed on an ABI 7500 Real-time PCR system (Applied Biosystems, Carlsbad, CA, USA). The PCR conditions were $30 \mathrm{sec}$ at $95^{\circ} \mathrm{C}$ followed by 45 cycles of $95^{\circ} \mathrm{C}$ for $5 \mathrm{sec}$ and $60^{\circ} \mathrm{C}$ for $34 \mathrm{sec}$. All of the quantitative PCR reactions were run in triplicate, and data were analyzed according to the comparative $\mathrm{Ct}\left(2^{-\Delta \Delta \mathrm{Ct}}\right)$ method. The qPCR primers (Table I) were synthesized by Sangon Biotech (Shanghai, China). Experiments were carried out independently three times.

Western blot analysis. Whole cell extracts were prepared by homogenizing cells in a lysis buffer [50 mM Tris (pH 7.2), $500 \mathrm{mM} \mathrm{NaCl}, 1 \%$ Triton X-100, $0.5 \%$ sodium deoxycholate, $0.1 \%$ SDS, $10 \mathrm{mM} \mathrm{MgCl}{ }_{2}$ with $10 \mu \mathrm{g} / \mathrm{ml}$ leupeptin, $10 \mu \mathrm{g} / \mathrm{ml}$ aprotinin, and $1 \mathrm{mM}$ PMSF]. Protein lysates were quantified by the Bradford method. Proteins were separated by sodiumdodecyl sulfate polyacrylamide gel (12\%) electrophoresis, transferred onto PVDF membranes (Millipore, Bedford, MA, USA) and blocked with TBST supplemented with 5\% non-fat milk. The membranes were immunoblotted with primary antibodies: rabbit anti-S100A4 antibody (1:500 dilution; Abcam); rabbit anti-GDF15 antibody (1:1,000 dilution;
Table I. The primers used for qPCR analysis.

\begin{tabular}{ll}
\hline Gene & \multicolumn{1}{c}{ Primer sequence (5'-3') } \\
\hline S100A4 & F: CCCTGGATGTGATGGTGT \\
& R: GTTGTCCCTGTGCTGTC \\
PLK2 & F: GAGCAGCTGAGCACATCATT \\
& R: CATGTGAGCACCATTGTTGA \\
$M D M 2$ & F: GTGAAGGAAACTGGGGAGTCTT \\
& R: AGGTACAGACATTTGGTATTGCA \\
$M T 1 G$ & F: CTTCTCGCTTGGGAACTCTA \\
& R: AGGGGTCAAGATTGTAGCAAA \\
TSP-1 & F: CGGAAAGAGTTAAGTGTCTAACAAA \\
& R: TCCTTATTGGGATACTTCTCTGC \\
STMN3 & F: GTCCCACAAAAGCCAGATGT \\
& R: ACCAAGACAGCCCAGAAG \\
GDF15 & F: CTCCAGATTCCGAGAGTTC \\
& R: AGAGATACGCAGGTGCAGGT \\
GDF15 & F: AGCTGTGGTCATTGGAGTGTT \\
promoter & R: TTCACCGTCCTGAGTTCTTGC \\
$G A P D H$ & F: ATCATCAGCAATGCCTCC \\
& R: CATCACGCCACAGTTTCC
\end{tabular}

F, forward; R, reverse.

ImmunoWay); and rabbit anti- $\beta$-actin antibody (1:500 dilution; Santa Cruz). After washing, membranes were incubated with a peroxidase-conjugated second antibody: mouse anti-rabbit $\mathrm{IgG}$ for S100A4 or GDF15 and $\beta$-actin. Immunoreactivity was detected using an enhanced chemiluminescence reagent (Amersham Biosciences, Freiburg, Germany) and visualized with Micro Chemi (DNR Bio-Imaging Systems, Jerusalem, Israel). Experiments were carried out independently three times.

Microarray analysis. Total RNA from the MGC803/ S100A4-siRNA cells or MGC803/NC-siRNA cells was extracted, cleaned up, reverse-transcribed, and hybridized to the Genechip ${ }^{\circledR}$ PrimeView $^{\mathrm{TM}}$ Human Gene Expression array (Affymetrix, Santa Clara, CA, USA) by the GeneChem Co. (Shanghai, China). Fold changes in expression and P-values were calculated from the raw data. Genes showing significant differential expression were defined as those exhibiting changes in expression exceeding 1.5 -fold with a P-value of $<0.05$; the differentially expressed gene transcripts were then included in further analyses. Briefly, gene transcripts showing significant changes in expression in the transcriptome array analysis were mapped to their corresponding Kyoto Encyclopedia of Genes and Genomes (KEGG) pathways and Gene Ontology (GO) molecular functions.

Chromatin immunoprecipitation (ChIP) assay. ChIP assays were performed using ChIP-IT ${ }^{\circledast}$ Express Enzymatic kits (Active Motif) according to the manufacturer's instructions. Briefly, $4.5 \times 10^{7}$ MGC803 cells were cross-linked with 


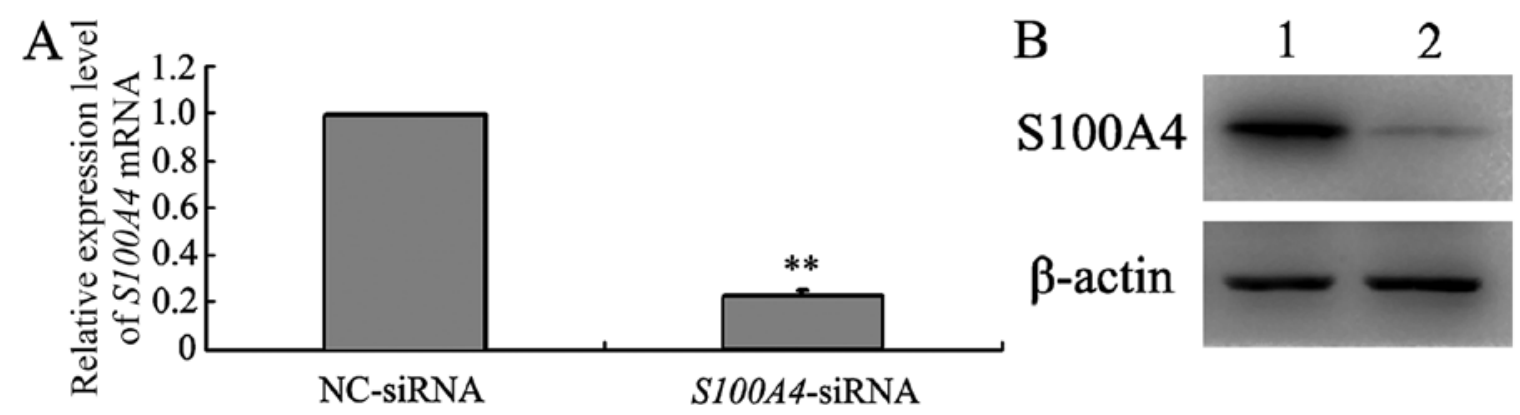

Figure 1. Downregulation of S100A4 expression in gastric cancer cells by RNAi. MGC803 cells transfected with either S100A4-siRNA or NC-siRNA were grown under normal culture conditions. mRNA and protein were extracted for qRT-PCR (A) and western blot (B) analyses, respectively, as described in Materials and methods. (A) Data represent the mean \pm SD from three experiments ( $\left.{ }^{* *} \mathrm{P}<0.01\right)$. (B) Lanes 1 and 2, MGC803/NC-siRNA cells and MGC803/ S100A4-siRNA cells, respectively, at $48 \mathrm{~h}$ after transfection.

$60 \mathrm{ml} 1 \%$ formaldehyde for $10 \mathrm{~min}$ at $37^{\circ} \mathrm{C}$. The cells were then resuspended in $3 \mathrm{ml}$ lysis buffer containing $15 \mu \mathrm{l} \mathrm{PIC}$ and $15 \mu \mathrm{l}$ PMSF and incubated for $45 \mathrm{~min}$ on ice. Fragments (150-450 bp) were generated by enzymatic shearing. The recovered supernatant $(400 \mu \mathrm{l})$ was incubated with $10 \mu \mathrm{g}$ antiS100A4 antibody (Abcam) or $10 \mu \mathrm{g}$ normal isotype control $\mathrm{IgG}$ and $25 \mu \mathrm{l}$ magnetic beads overnight at $4^{\circ} \mathrm{C}$ with rotation. Approximately $200 \mu \mathrm{l}$ of the recovered supernatant was used as the input. After washing the magnetic beads/antibody/DNA complex, crosslinking was reversed by incubation with $10 \mu \mathrm{l}$ $5 \mathrm{M} \mathrm{NaCl}$ at $65^{\circ} \mathrm{C}$ for $8 \mathrm{~h}$. The DNA samples were then purified and analyzed by quantitative polymerase chain reaction (qPCR) using the primers listed in Table I. Experiments were carried out independently three times.

Construction and transfection of the GDF15 expression vector. GV141-GDF15, the expression vector specific for human GDF15 was constructed by GeneChem. GV141-empty (negative control) was provided by GeneChem. MGC803 cells were transfected with these vectors using Lipofectamine ${ }^{\mathrm{TM}} 2000$ transfection reagent (Invitrogen) according to the manufacturer's instructions. The cells transfected with GV141-GDF15 or GV141-empty were referred to as MGC803/GV141-GDF15 cells or MGC803/ GV141-empty cells, respectively. Cells were harvested at $48 \mathrm{~h}$ after transfection for the associated experiments.

Spheroid formation assay. Single cell suspensions of transfected MGC803 cells were plated ( $1 \times 10^{3}$ cells/well) in 24-well Ultra-Low Attachment Plates (Corning) and maintained in serum-free DMEM/F-12 medium supplemented with $20 \mathrm{ng} / \mathrm{ml}$ basic fibroblast growth factor, $20 \mathrm{ng} / \mathrm{ml}$ epidermal growth factor, $10 \mathrm{mmol} / 1 \mathrm{HEPES}, 0.4 \%$ bovine serum albumin and B27 supplement (1:50 dilution; Invitrogen) for 7 days. The number of spheroids (diameter $>75 \mu \mathrm{m}$ ) was counted and representative images were captured under an inverted microscope (Olympus, Tokyo, Japan). Experiments were carried out independently three times.

Soft-agar colony formation assay. Single cell suspensions of transfected MGC803 cells were plated into 6-well plates $\left(3 \times 10^{3}\right.$ cells/well) in RPMI-1640 (Invitrogen) containing $10 \% \mathrm{FBS}$ and $0.3 \%$ low melting-point agarose (Amresco, Solon, OH, USA) on a base layer of $0.5 \%$ low melting-point agarose. After incubation for 7 days at $37^{\circ} \mathrm{C}$, the number of colonies $>50 \mu \mathrm{m}$ was counted and photographed. Experiments were carried out independently three times.

Rescue assay after co-transfection of S100A4-siRNA and GV141-GDF15. S100A4-siRNA and the GV141-GDF15 vector were co-transfected into MGC803 cells using Lipofectamine ${ }^{\mathrm{TM}}$ 2000 (Invitrogen) to generate MGC803/S100A4-siRNA+ GV141-GDF15 cells. S100A4-siRNA and GV141-empty vector were co-transfected to generate MGC803/S100A4-siRNA+ GV141-empty cells as a control. At $48 \mathrm{~h}$ after transfection, cells were harvested for investigation of CSC-like properties by spheroid formation assays and soft-agar colony formation assays. Experiments were carried out independently three times.

Statistical analysis. Statistical analysis was carried out by Student's t-test using the Statistical Package for the Social Sciences (SPSS Inc., Chicago, IL, USA), where $\mathrm{P}<0.05$ was considered to indicate statistical significance.

\section{Results}

Knockdown of S100A4 expression in MGC803 cells by RNA interference (RNAi). The effect of S100A4-siRNA transfection on S100A4 gene silencing was evaluated by both qRT-PCR and western blot analyses. As shown in Fig. 1, endogenous S100A4 mRNA and protein levels were reduced in MGC803/ S100A4-siRNA cells at $48 \mathrm{~h}$ post-transfection compared with those in MGC803/NC-siRNA cells. There was no significant difference in $\beta$-actin expression between the two groups. These data indicate that $5100 A 4$-siRNA effectively suppressed S100A4 expression in MGC803 cells.

Gene expression profiling in MGC803 cells after S100A4 silencing. Alterations in the expression of the MGC803 cell transcriptome associated with S100A4 knockdown were analyzed by cDNA microarray profiling. Compared with the profile of MGC803/NC-siRNA cells, differential expression of 179 transcripts was identified in MGC803/S100A4-siRNA cells ( $>1.5$-fold change in expression; $\mathrm{P}<0.05)$ (Fig. 2). Of the 179 differentially expressed genes (DEGs), 38 were upregulated and 141 were downregulated in S100A4-silenced cells (data not shown in detail). 


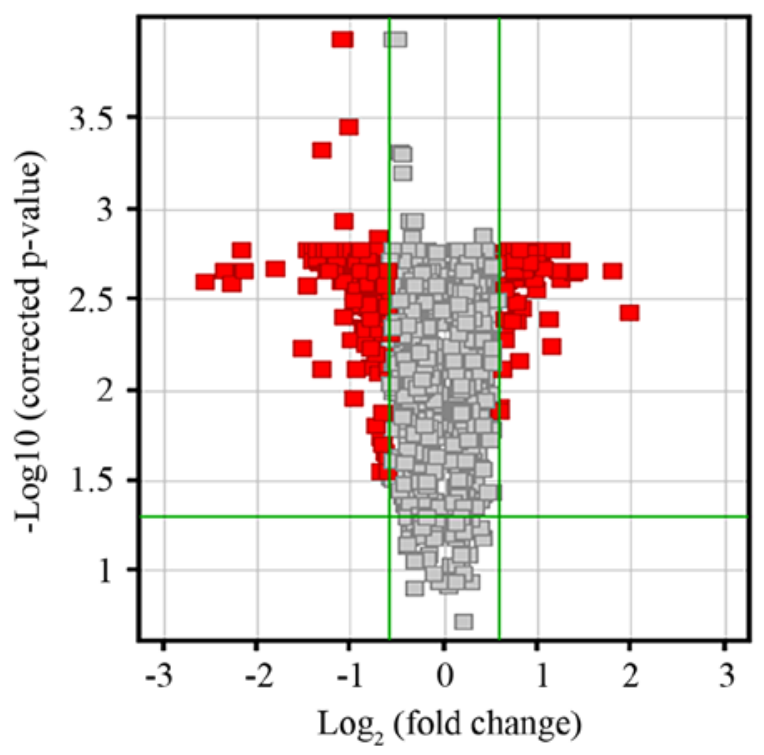

Figure 2. Volcano plot analysis of differentially expressed genes identified in cDNA microarray analysis. The expression of 179 genes was significantly changed ( $>1.5$-fold) in MGC803/S100A4-siRNA cells at $48 \mathrm{~h}$ after transfection compared to the MGC803/NC-siRNA cells $(\mathrm{P}<0.05)$. A total of 38 genes were upregulated and 141 were downregulated. $\mathrm{x}$-axis parallel lines, $\mathrm{P}$-value, 0.05 . $\mathrm{y}$-axis parallel lines, fold change $=1.5$. Red areas indicate significant changes in gene expression.

Table II. Functional enrichment of GO analysis following S100A4 knockdown in gastric cancer cells.

\begin{tabular}{c}
\hline Molecular function \\
Transferase activity \\
Kinase activity \\
Receptor binding \\
Actin filament binding \\
Hydrogen ion transmembrane transporter activity \\
Enzyme regulator activity \\
Phosphotransferase activity \\
Receptor activity \\
Monovalent inorganic cation transmembrane transporter activity \\
Protease inhibitor activity
\end{tabular}

Table III. Functional enrichment of KEGG pathway analysis following S100A4 knockdown in gastric cancer cells.

\begin{tabular}{c}
\hline KEGG pathway \\
p53 signaling pathway \\
Bladder cancer \\
Glutathione metabolism \\
CCR5 pathway \\
Focal adhesion \\
Complement and coagulation cascades \\
ECM receptor interaction \\
Prostate cancer \\
Sasopressin regulated water reabsorption \\
SARS pathway
\end{tabular}

Gene ontology term and KEGG pathway enrichment analyses. The molecular functions involving DEGs were identified by Gene Ontology (GO) enrichment analysis. In total, $10 \mathrm{GO}$ terms were identified (Table II). To identify well-characterized pathways that were significantly represented, the list of genes was also subjected to Kyoto Encyclopedia of Genes and Genomes (KEGG) pathway analysis. Ten significantly enriched pathways were identified (Table III), although the vast majority of the 179 DEGs could not be assigned to these signaling pathways or molecular classifications.

Validation of microarray data by $q R T-P C R$ analysis. The microarray data were validated by qRT-PCR analysis of RNA from the same cell samples. As shown in Fig. 3, the expression profiles of the six genes selected from the 179 DEGs were consistent with those determined in the microarray analysis; thus, validating the accuracy of the microarray data. Among the DEGs, expression of the growth differentiation factor-15 gene $(G D F 15)$ was shown to be downregulated by 5.11- and 8.18-fold by microarray and qRT-PCR analyses, respectively. Thus, GDF15 was identified as one of the most downregulated genes in MGC803 cells following S100A4 inhibition using both these techniques.

GDF15 is an important downstream gene of S100A4. GDF15 protein expression in MGC803 cells after S100A4 inhibition

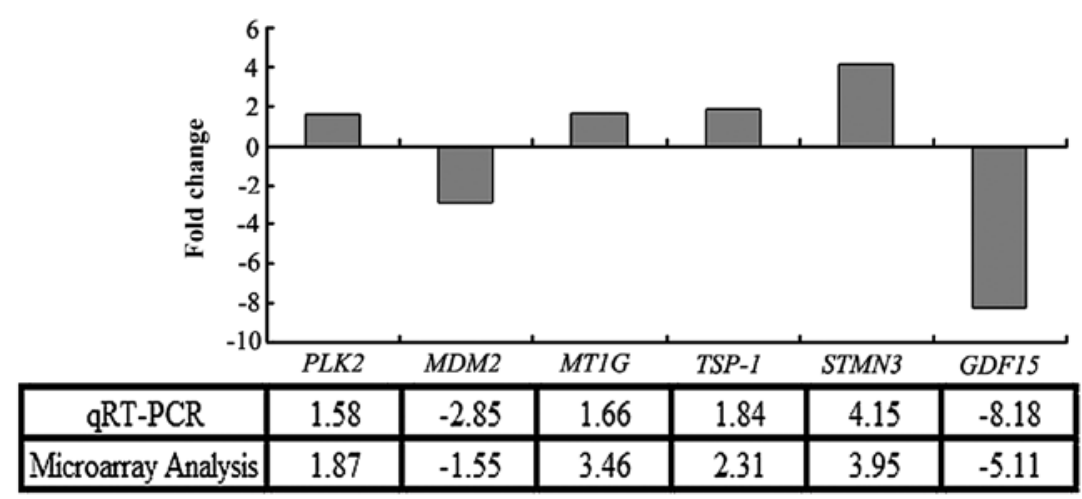

Figure 3. Validation of microarray data by qRT-PCR. The expression of six differentially expressed genes identified in microarray analysis was detected by qRT-PCR. Data represent the mean of three experiments, normalized to GAPDH, and presented as fold change in expression in MGC803/S100A4-siRNA cells relative to that in MGC803/NC-siRNA cells. 


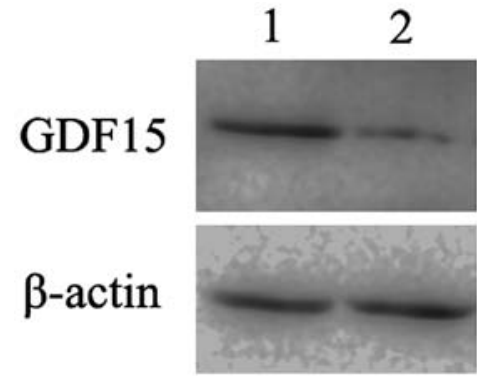

Figure 4. The effect of S100A4 inhibition on GDF15 protein expression. GDF15 protein levels were detected by western blot analysis after $S 100 A 4$ inhibition. Lanes 1 and 2, MGC803/NC-siRNA cells and MGC803/S100A4siRNA cells, respectively, at $48 \mathrm{~h}$ after transfection.

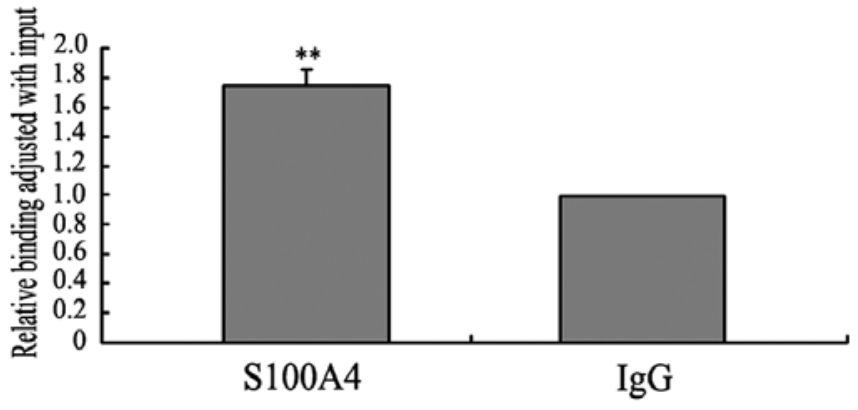

Figure 5. ChIP assays indicated that S100A4 binds to the GDF15 promoter in vivo. ChIP assays were performed in MGC803 cells using an antibody directed against $5100 A 4$ or an IgG control. ChIP-derived DNA was amplified by qPCR with specific primers designed to amplify the proximal promoter region (-113 bp to +61 ) of $G D F 15$. Data are shown relative to $\mathrm{qPCR}$ products amplified with input DNA. Data represent the mean \pm SD from three experiments $\left({ }^{* *} \mathrm{P}<0.01\right)$

was also determined by western blot analysis. S100A4-siRNA transfection induced a substantial decrease in GDF15 protein expression in MGC803 cells (Fig. 4), further confirming that GDF 15 is an important downstream gene of S100A4 and subject to positive regulation.

ChIP analysis of S100A4 binding to the GDF15 promoter. To determine whether S100A4 binds to the GDF15 promoter in vivo, we performed chromatin immunoprecipitation (ChIP) and then analyzed the quantity of DNA fragments flanking the proximal promoter region $(-113$ to $+61 \mathrm{bp})$ of GDF 15 by qPCR. The qPCR analysis showed that the quantity of DNA fragments derived from immunoprecipitation with the anti-S100A4 antibody was almost 1.7-fold higher than that derived from immunoprecipitation with the $\mathrm{IgG}$ antibody (Fig. 5). These results suggested that S100A4 might bind to the GDF15 promoter in vivo, and therefore implicates S100A4 in the regulation of $G D F 15$ expression at the transcriptional level.

GDF15 overexpression promotes the CSC-like properties of MGC803 cells. Compared with GV141-empty vector transfection, GV141-GDF15 transfection into MGC803 cells led to increased GDF15 expression at both the mRNA and protein levels (Fig. 6). We then investigated the effects of GDF15 on the CSC-like properties of MGC803 cells by performing spheroid formation and soft-agar colony formation assays. More spheroids and colonies were observed in MGC803/ GV141-GDF15 cells than in MGC803/GV141-empty cells (Fig. 7), suggesting that GDF15 overexpression promotes the CSC-like properties of MGC803 cells.

GDF 15 mediates the effects of S100A4 on CSC-like properties of MGC803 cells. We first explored the effects of S100A4 inhibition on CSC-like properties of MGC803 cells. MGC803/S100A4-siRNA cells formed fewer spheroids and colonies than MGC803/NC-siRNA cells, which suggested that S100A4 inhibition decreased the CSC-like properties of MGC803 cells (Fig. 8). To corroborate the role of GDF15 in S100A4-regulated CSC-like properties, we carried out rescue experiments by co-transfection of S100A4-siRNA with GV141-GDF15 or GV141-empty into MGC803 cells. qRT-PCR and western blot analyses showed that compared to MGC803/ S100A4-siRNA+GV141-empty cells, MGC803/S100A4siRNA+GV141-GDF15 cells displayed increased expression of $G D F 15$ at $48 \mathrm{~h}$ after transfection. This indicated that the GV141-GDF15 vector transfection reversed the downregulation of GDF15 caused by S100A4 inhibition (Fig. 9). Furthermore, compared with MGC803/S100A4-siRNA+GV14-empty cells,

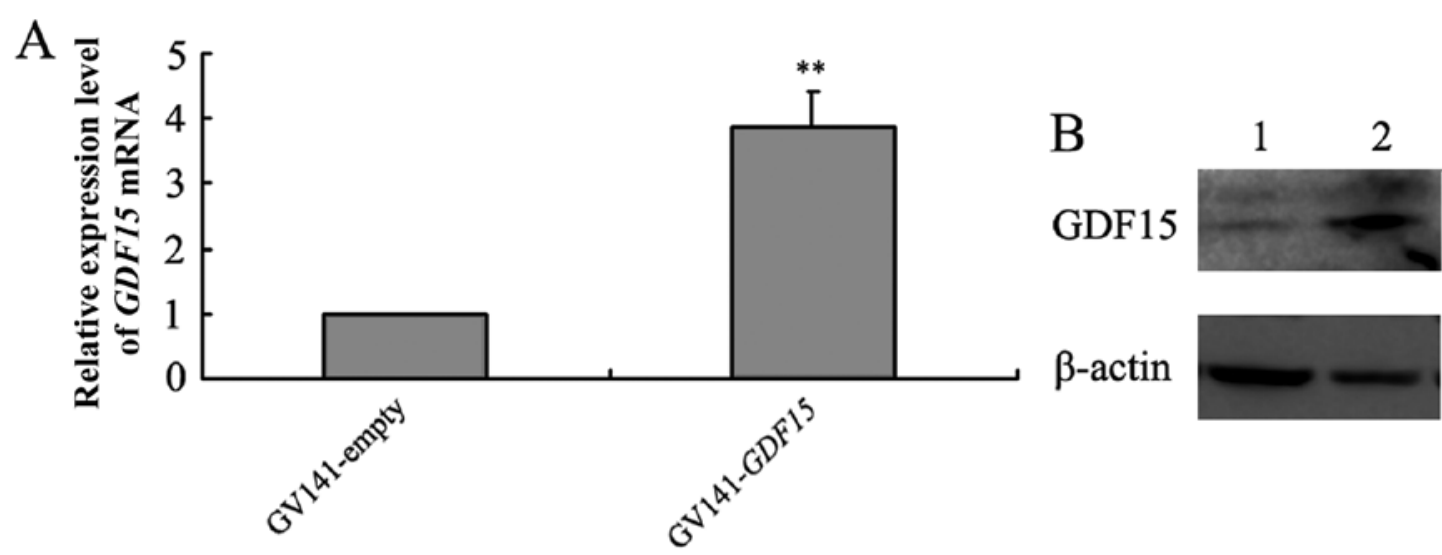

Figure 6. GV141-GDF15 transfection enhances GDF15 expression in MGC803 cells. Expression of GDF15 in MGC803/GV141-GDF15 cells or MGC803/ GV141-empty cells was analyzed by qRT-PCR (A) and western blotting (B), respectively, as described in Materials and methods. (A) Data represent the mean \pm SD from three experiments ( $\left.{ }^{* *} \mathrm{P}<0.01\right)$. (B) Lanes 1 and 2, GV141-empty vector and GV141-GDF15 transfection, respectively, at $48 \mathrm{~h}$ after transfection. 


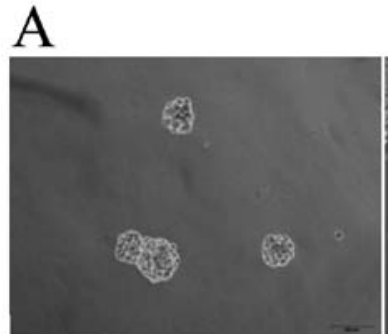

GV141-empty

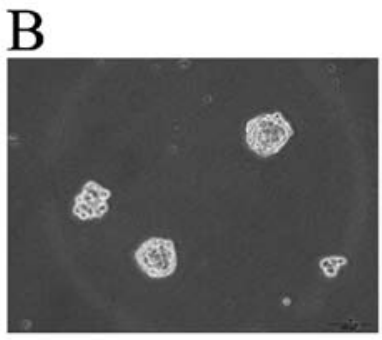

GV141-empty

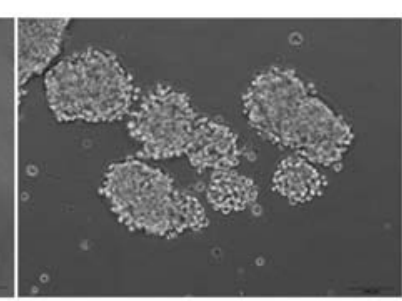

GV141-GDF15

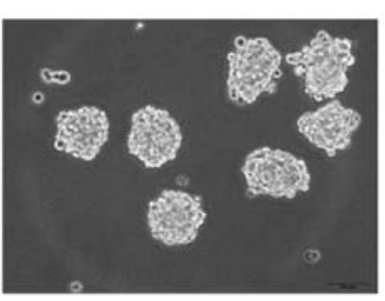

GV141-GDF15
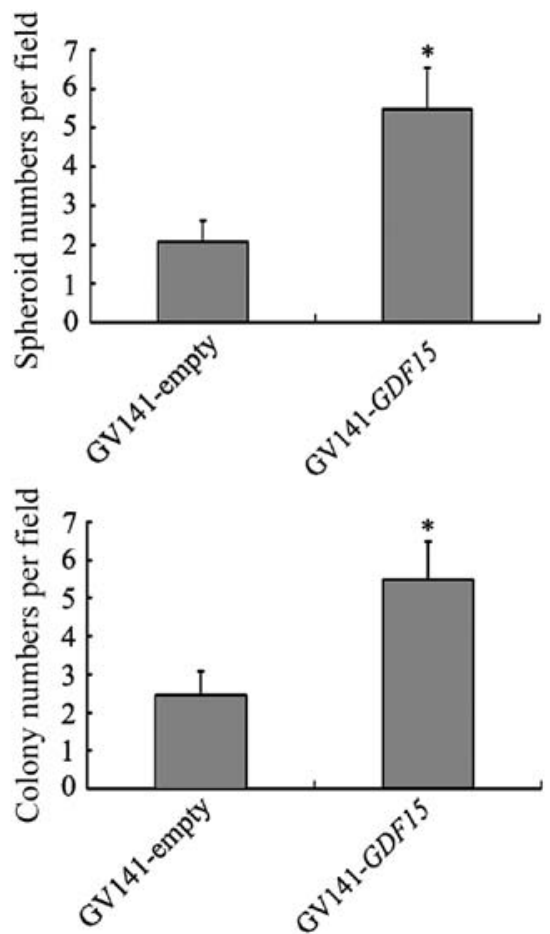

Figure 7. The effects of GDF15 on the CSC-like properties of MGC803 cells. (A) Representative images of spheroids formed by MGC803/GV141-empty or MGC803/GV141-GDF15 cells (left, magnification, x20). Bar graph (right) represents the mean number of spheroids from five randomly selected fields under the microscope, and error bars represent SD, ${ }^{*} \mathrm{P}<0.05$. (B) Representative images of colonies formed in soft-agar by MGC803/GV141-empty or MGC803/ GV141-GDF15 cells (left, magnification, x20). Bar graph (right) represents the mean number of colonies from five randomly selected fields under the microscope, and error bars represent $\mathrm{SD},{ }^{*} \mathrm{P}<0.05$. All the results were obtained from three experiments.

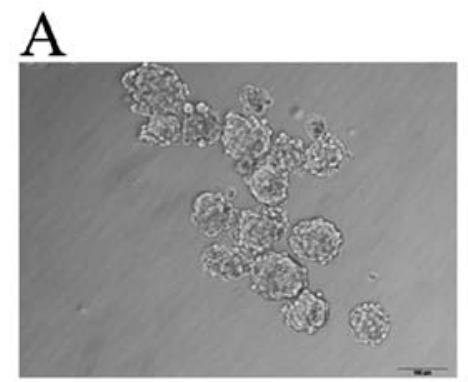

NC-siRNA

$\mathrm{B}$

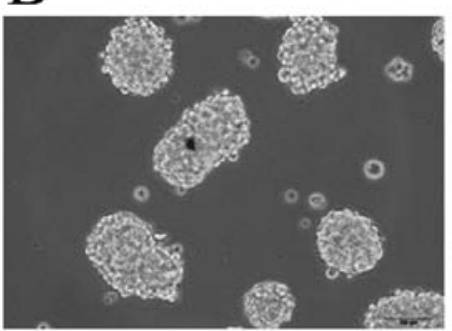

NC-siRNA

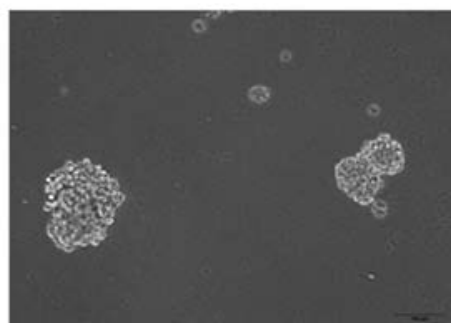

S100A4-siRNA

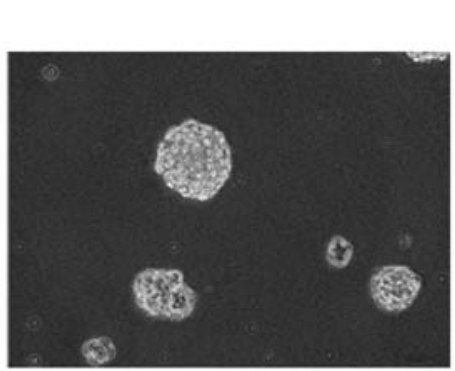

S100A4-SiRNA
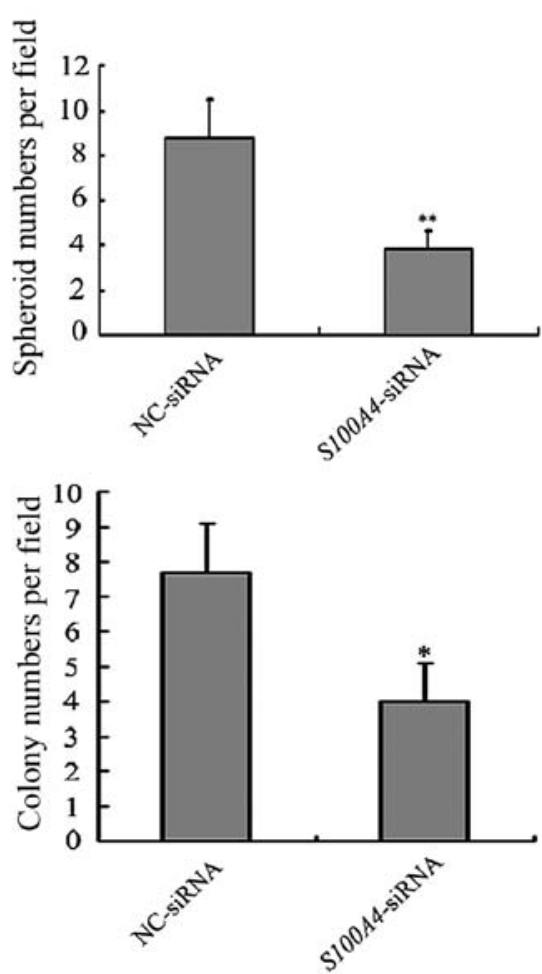

Figure 8. S100A4-siRNA transfection inhibits the spheroid and soft-agar colony forming abilities of MGC803 cells. (A) Detection of spheroid forming ability: representative images of spheroids formed by MGC803/NC-siRNA or MGC803/S100A4-siRNA cells (left, magnification, x20). Bar graph (right) represents the mean number of spheroids from five randomly selected fields under the microscope, and error bars represent $S D$, ${ }^{* *} \mathrm{P}<0.01$. (B) Detection of colony forming ability: representative images of colonies formed by MGC803/NC-siRNA or MGC803/S100A4-siRNA cells (left, magnification, x20). Bar graph (right) represents the mean number of colonies from five randomly selected fields under the microscope, and error bars represent $\mathrm{SD}$, ${ }^{*} \mathrm{P}<0.05$. All the results were obtained from three experiments. 


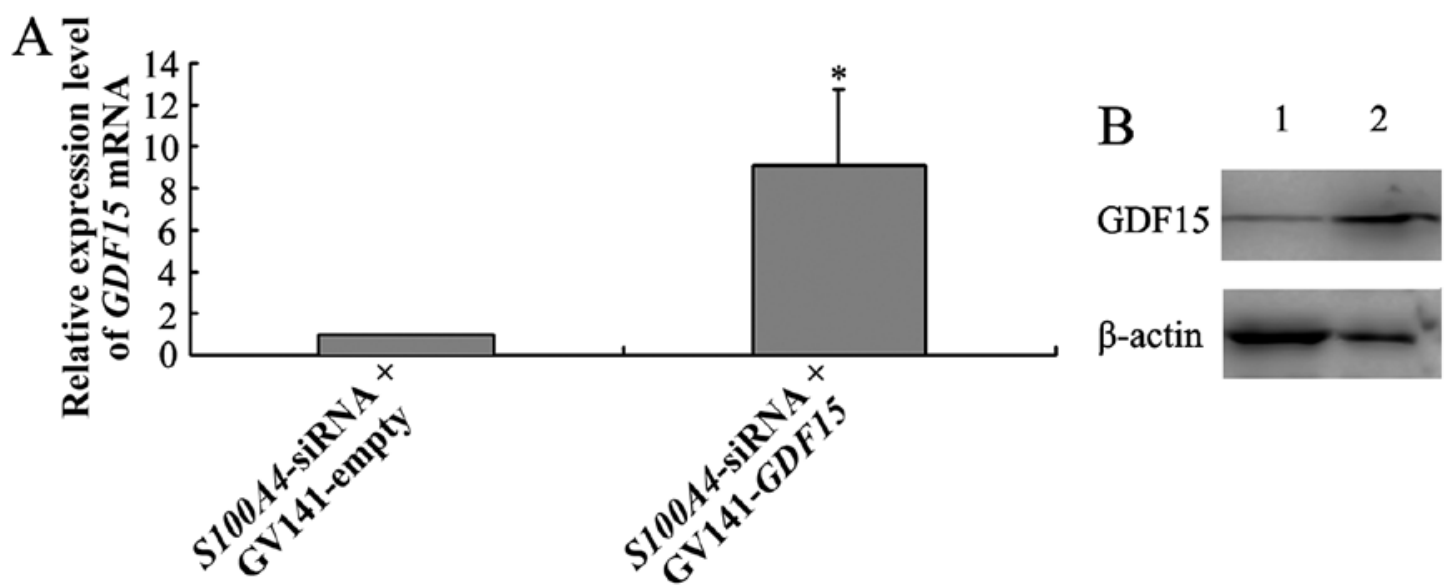

Figure 9. GV141-GDF15 vector transfection reverses the downregulation of GDF15 by S100A4 inhibition. MGC803 cells were co-transfected with S100A4siRNA and either GV141-empty vector or GV141-GDF15 vector. GDF15 expression in the co-transfected cells was analyzed by qRT-PCR (A) and western blotting (B), respectively, as described in Materials and methods. (A) Data represent the mean \pm SD from three experiments ("P<0.05). (B) Lanes 1 and 2, expression of GDF15 in MGC803 cells at $48 \mathrm{~h}$ after co-transfection of S100A4-siRNA with either GV141-empty vector or GV141-GDF15 vector, respectively.

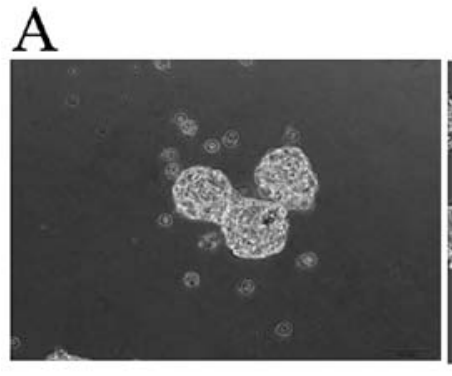

S100A4-siRNA+GV141-empty

B

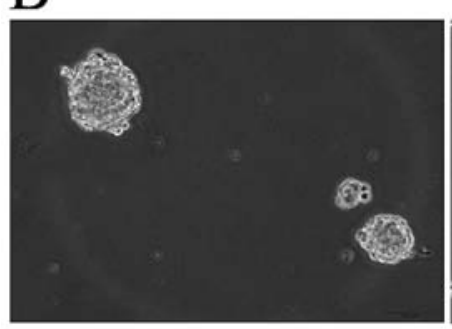

S100A4-siRNA+GV141-empty

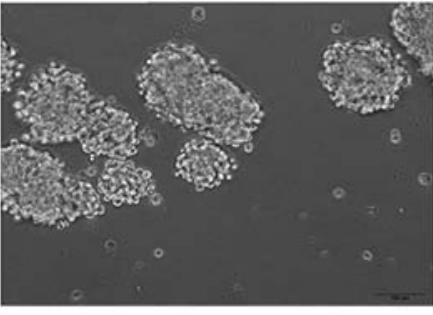

S100A4-SiRNA+GV141-GDF15
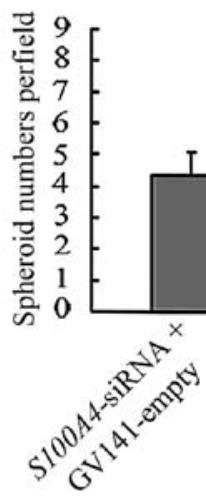

$\times$
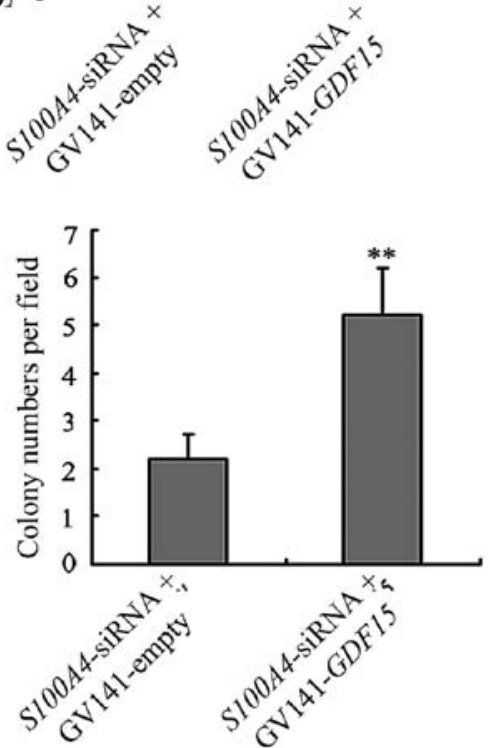

Figure 10. GV141-GDF15 vector transfection reverses the effects of S100A4 inhibition on the spheroid and soft-agar colony forming abilities of MGC803 cells. (A) Detection of spheroid forming ability: representative images of spheroids formed by MGC803/S100A4-siRNA+GV141-empty and MGC803/S100A4siRNA+GV141-GDF15 cells (left, magnification, x20). Bar graph (right) represents the mean number of spheroids from five randomly selected fields under the microscope, and error bars represent SD, ${ }^{*} \mathrm{P}<0.05$. (B) Detection of colony forming ability: representative images of colonies formed by MGC803/S100A4siRNA+GV141-empty and MGC803/S100A4-siRNA+GV141-GDF15 cells (left, magnification, x20). Bar graph (right) represents the mean number of colonies from five randomly selected fields under the microscope, and error bars represent $\mathrm{SD},{ }^{* *} \mathrm{P}<0.01$. All the results were obtained from three repeat experiments.

significantly more spheroids and colonies were formed by MGC803/S100A4-siRNA+GV14-GDF15 cells, indicating that GDF15 mediates the effects of S100A4 on the CSC-like properties of MGC803 cells (Fig. 10).

\section{Discussion}

Many studies have shown S100A4 overexpression in various human cancers, such as hepatocellular, clear cell renal cell 
and gastric cancers, and that it is closely related to metastasis and poor patient prognosis (15-17). Previously, our group demonstrated that reducing S100A4 expression altered cell proliferation, apoptosis, migration and anoikis in BGC823 cells in vitro, and inhibited xenograft tumor growth in vivo $(8,18,19)$. This led us to suggest that $\$ 100 A 4$ influences key cellular processes associated with the progression of gastric cancer. To gain insight into the mechanisms underlying this process, we first performed cDNA microarray analysis of the global alterations in gene expression in MGC803 gastric cancer cells following siRNA-mediated S100A4 inhibition. Among the total genes investigated, 179 DEGs (38 upregulated and 141 downregulated) were identified in S100A4-siRNA transfected MGC803 cells compared with those transfected with NC-siRNA treated cells. In recent years, studies have shown that S100A4 inhibition leads to changes in the expression of many genes in various tumor cells. In a study of the mechanism by which S100A4 gene influences the invasiveness of prostate cancer cells using a microarray containing 96 well-characterized metastatic genes, Saleem et al (20) found that many genes, including matrix metalloproteinase 9 $(M M P-9)$ and its tissue inhibitor (TIMP-1), were highly responsive to $S 100 A 4$ gene suppression. Using metastasisrelated gene mRNA microarrays, Huang et al (21) identified some significantly dysregulated genes after downregulation of S100A4, including three downregulated genes (MMP-9, $M M P-10$ and $C D H 11)$ and one upregulated gene (TIMP-4) in human colorectal cancer cells. Ochiya et al (22) reported that RNAi-mediated S100A4 knockdown in mouse endothelial MSS31 cells markedly suppressed in vitro capillary-like tube formation in the early stages after treatment. Furthermore, this effect was found to be associated with down- and upregulation of the expression of some pro-angiogenic (aqpl, fgfl8, retn, map3k5, thyl, foxo6, hs6stl and $\mathrm{mmp}-3$ ) and anti-angiogenic (cdknla, thbsl and spry4) genes. These results indicated that S100A4 influences the expression of many downstream genes in different kinds of cells. To the best of our knowledge, this study is the first to investigate the DEG profile downstream of S100A4 in gastric cancer cells. The results provide important and extensive information for the clarification of the mechanism by which S100A4 influences the progression of gastric cancer.

We undertook further bioinformatics analysis of the 179 DEGs to investigate the functional relevance of these genes. Pathway analysis showed the involvement of many of the 179 DEGs in 10 pathways, including the p53 signaling pathway, focal adhesion, ECM receptor interactions and others. GO annotation analysis revealed that DEGs were related to 10 types of molecular functions, including transferase activity, kinase activity, receptor binding and others. Nevertheless, a large number of the 179 DEGs were not associated with these pathways or molecular functions and their functions should not be ignored. In combination, our findings indicate that S100A4 participates in a variety of pathways and influences many types of molecular functions by regulating downstream gene expression in gastric cancer cells.

Growth differentiation factor-15 (GDF15), also known as MIC-1, PTGFB and PLAB (23-25), is a divergent member of the TGF- $\beta$ superfamily $(26,27)$. Although normally undetectable under physiologic conditions in any tissues except the placenta (28), GDF15 becomes highly upregulated under some pathological conditions, such as cancer, myocardial infarction and inflammation (29). GDF15 has been shown to be a marker of mortality with high serum levels being a predictor of death, particularly due to cancer (30). Elevated circulating GDF15 levels may correlate with poor clinical outcomes in endometrial cancer and can be used as a biomarker of the endometrial cancer phenotype, including the presence of lymph node metastasis and reduced survival (31). In addition, GDF15 exerts an antiapoptotic effect on oral squamous cell carcinoma cells in vitro (32). Tsui et al (33) demonstrated that GDF15 overexpression induces cell proliferation, invasion, and tumorigenesis of $\mathrm{PC}-3$ prostate carcinoma cells. All these results indicate that GDF15 plays important roles in many types of cancers. Furthermore, in this study, GDF15 was found to be one of the most notable DEGs, with downregulated expression after S100A4 inhibition exceeding 5-fold identified in microarray analysis and 8-fold in the qRT-PCR analysis. These results indicate that GDF15 is an important downstream gene of S100A4 and is upregulated by it; consequently, we focused on GDF15 in further research. First, we investigated the effects of S100A4 on GDF15 expression in gastric cancer cells. Previous report showed that S100A4 protein could interact with p53 protein and influence the expression of p53 target genes, such as TSP-1 and MDM2 in CSML-0 murine non-metastatic adenocarcinoma cells (34). Furthermore, cDNA microarray analysis in this study also showed differential expression of these genes after S100A4 inhibition in gastric cancer MGC803 cells, which indicated that S100A4 may also affect the expression of p53 target genes in MGC803 cells. Recently, it has been reported that GDF15 is a direct target of p53 (35). ChIP analysis performed in this study to explore the mechanisms by which $S 100 A 4$ regulates $G D F 15$ expression revealed that $\mathrm{S} 100 \mathrm{~A} 4$ protein binds to the proximal promoter region of $G D F 15$, which contains the p53 binding sites as reported (36). We speculated that S100A4 functions as a co-factor, interacting with p53 to regulate $G D F 15$ expression at the transcriptional level in MGC803 cells. In addition, the proximal promoter region of GDF15 investigated in our ChIP analysis contains binding sites for other transcription factors such as Sp1 (37) and EGR-1 (38). Thus, it can be speculated that S100A4 may also affect the expression of GDF15 by cooperating with Sp1, EGR-1 or other transcriptional factors. However, the underlying mechanism remains to be elucidated.

Next, we investigated the functional significance of $G D F 15$ in gastric cancer. It has been reported that GDF15 is upregulated at the transcriptional level in tissues and cell lines of gastric cancers and GDF15 increased the invasiveness of gastric cancer cells through regulation of urokinase plasminogen activator $(39,40)$. In addition, studies have shown that serum levels of GDF15 and MMP-7 have diagnostic value for gastric cancers. The combination marker formed by GDF15, MMP-7 and miR-200c is indicative of adverse evolution in gastric cancer patients (41). However, the role of GDF15 in gastric cancer is far from clear. Recent studies show that GDF15 can enhance the tumor-initiating and self-renewal potential of multiple myeloma cells (42). Therefore, we speculated that GDF15 might affect CSC-like properties of gastric cancer cells. To investigate this, we analyzed the CSC-like properties of MGC803 cells transfected with a GDF15 expression vector. We found that the numbers of spheroids and colonies 
were increased significantly after $G D F 15$ expression vector transfection, indicating that GDF15 promotes the CSC-like properties of MGC803 cells.

Our previous findings showed that IL-1 $\beta$ regulates spheroid and soft-agar colony forming abilities of MGC803 cells through $S 100 A 4$, suggesting that S100A4 might be involved in the regulation of CSC-like properties (unpublished data). In this study, we showed that S100A4 inhibition led to decreased spheroid and soft-agar colony forming abilities of MGC803 cells, demonstrating that $S 100 A 4$ promotes the CSC-like properties of MGC803 cells.

Based on these findings, we speculated that GDF15 might influence the effects of S100A4 on the CSC-like properties in MGC803 gastric cancer cells. To test this hypothesis, we carried out rescue experiments by co-transfection of S100A4-siRNA with GV141-GDF15 or GV141-empty into MGC803 cells. The results showed that GV141-GDF15 vector transfection reversed the reduced spheroid and soft-agar colony forming abilities induced by $S 100 A 4$ inhibition. These findings suggest that, as a downstream effector, GDF15 at least partly mediates $S 100 A 4$ regulation of CSC-like properties in MGC803 cells.

In conclusion, we conducted the first expression profile analysis of S100A4 downstream genes in MGC803 gastric cancer cells, followed by experimental validation and functional analysis. Our results suggest that S100A4 influences the expression of many genes in gastric cancer cells. S100A4 can bind to the GDF15 promoter and may regulate its expression at the transcriptional level. We also provide experimental evidence suggesting that GDF15 promotes the CSC-like properties of MGC803 gastric cancer cells, and that S100A4 influences this effect by regulating GDF15 expression. These data provide a novel overview of the effect of S100A4 on the expression of downstream genes, and an insight into the mechanisms by which S100A4 influences the CSC-like properties of MGC803 gastric cancer cells.

\section{Acknowledgements}

This study was supported by the National Natural Science Foundation of China; contract grant no. 81272717.

\section{References}

1. Boye K and Maelandsmo GM: S100A4 and metastasis: A small actor playing many roles. Am J Pathol 176: 528-535, 2010.

2. Natarajan J, Hunter K, Mutalik VS and Radhakrishnan R: Overexpression of S100A4 as a biomarker of metastasis and recurrence in oral squamous cell carcinoma. J Appl Oral Sci 22: 426-433, 2014.

3. Li H, Liu Z, Xu C, Chen Y, Zhang J, Cui B, Chen X, An G, She X, Liu H, et al: Overexpression of S100A4 is closely associated with the progression and prognosis of gastric cancer in young patients. Oncol Lett 5: 1485-1490, 2013.

4. Niu Y, Wang L, Cheng C, Du C, Lu X, Wang G and Liu J: Increased expressions of SATB1 and S100A4 are associated with poor prognosis in human colorectal carcinoma. APMIS 123 93-101, 2015.

5. Zhang K, Zhang M, Zhao H, Yan B, Zhang D and Liang J: S100A4 regulates motility and invasiveness of human esophageal squamous cell carcinoma through modulating the AKT/Slug signal pathway. Dis Esophagus 25: 731-739, 2012.

6. Wang L, Wang X, Liang Y, Diao X and Chen Q: S100A4 promotes invasion and angiogenesis in breast cancer MDA-MB-231 cells by upregulating matrix metalloproteinase-13. Acta Biochim Pol 59: $593-598,2012$
7. Che P, Yang Y, Han X, Hu M, Sellers JC, Londono-Joshi AI, Cai GQ, Buchsbaum DJ, Christein JD, Tang Q, et al: S100A4 promotes pancreatic cancer progression through a dual signaling pathway mediated by Src and focal adhesion kinase. Sci Rep 5: $8453,2015$.

8. Hua J, Chen D, Fu H, Zhang R, Shen W, Liu S, Sun K and Sun X: Short hairpin RNA-mediated inhibition of S100A4 promotes apoptosis and suppresses proliferation of BGC823 gastric cancer cells in vitro and in vivo. Cancer Lett 292: 41-47, 2010.

9. Clarke MF, Dick JE, Dirks PB, Eaves CJ, Jamieson $\mathrm{CH}$, Jones DL, Visvader J, Weissman IL and Wahl GM: Cancer stem cells - perspectives on current status and future directions: AACR Workshop on cancer stem cells. Cancer Res 66: 9339-9344, 2006.

10. Takaishi S, Okumura T, Tu S, Wang SS, Shibata W, Vigneshwaran R, Gordon SA, Shimada Y and Wang TC: Identification of gastric cancer stem cells using the cell surface marker CD44. Stem Cells 27: 1006-1020, 2009.

11. Larzabal L, El-Nikhely N, Redrado M, Seeger W, Savai R and Calvo A: Differential effects of drugs targeting cancer stem cell (CSC) and non-CSC populations on lung primary tumors and metastasis. PLoS One 8: e79798, 2013.

12. Ping YF and Bian XW: Consice review: Contribution of cancer stem cells to neovascularization. Stem Cells 29: 888-894, 2011.

13. Liao J, Qian F, Tchabo N, Mhawech-Fauceglia P, Beck A, Qian Z, Wang X, Huss WJ, Lele SB, Morrison CD, et al: Ovarian cancer spheroid cells with stem cell-like properties contribute to tumor generation, metastasis and chemotherapy resistance through hypoxia-resistant metabolism. PLoS One 9: e84941, 2014.

14. Lo JF, Yu CC, Chiou SH, Huang CY, Jan CI, Lin SC, Liu CJ, $\mathrm{Hu}$ WY and Yu YH: The epithelial-mesenchymal transition mediator S100A4 maintains cancer-initiating cells in head and neck cancers. Cancer Res 71: 1912-1923, 2011.

15. Liu Z, Liu H, Pan H, Du Q and Liang J: Clinicopathological significance of S100A4 expression in human hepatocellular carcinoma. J Int Med Res 41: 457-462, 2013.

16. Yang H, Zhao K, Yu Q, Wang X, Song Y and Li R: Evaluation of plasma and tissue S100A4 protein and mRNA levels as potential markers of metastasis and prognosis in clear cell renal cell carcinoma. J Int Med Res 40: 475-485, 2012.

17. Wang YY, Ye ZY, Zhao ZS, Tao HQ and Chu YQ: High-level expression of S100A4 correlates with lymph node metastasis and poor prognosis in patients with gastric cancer. Ann Surg Oncol 17: 89-97, 2010.

18. Shen W, Chen D, Fu H, Liu S, Sun K and Sun X: S100A4 protects gastric cancer cells from anoikis through regulation of $\alpha \mathrm{v}$ and $\alpha 5$ integrin. Cancer Sci 102: 1014-1018, 2011.

19. Chen D, Zhang R, Shen W, Fu H, Liu S, Sun K and Sun X: RPS12specific shRNA inhibits the proliferation, migration of BGC823 gastric cancer cells with S100A4 as a downstream effector. Int J Oncol 42: 1763-1769, 2013.

20. Saleem M, Kweon MH, Johnson JJ, Adhami VM, Elcheva I, Khan N, Bin Hafeez B, Bhat KM, Sarfaraz S, Reagan-Shaw S, et al: S100A4 accelerates tumorigenesis and invasion of human prostate cancer through the transcriptional regulation of matrix metalloproteinase 9. Proc Natl Acad Sci USA 103: 14825-14830, 2006

21. Huang L, Xu Y, Cai G, Guan Z and Cai S: Downregulation of S100A4 expression by RNA interference suppresses cell growth and invasion in human colorectal cancer cells. Oncol Rep 27: 917-922, 2012.

22. Ochiya T, Takenaga K and Endo H: Silencing of S100A4, a metastasis-associated protein, in endothelial cells inhibits tumor angiogenesis and growth. Angiogenesis 17: 17-26, 2014.

23. Bootcov MR, Bauskin AR, Valenzuela SM, Moore AG, Bansal M, He XY, Zhang HP, Donnellan M, Mahler S, Pryor K, et al: MIC-1, a novel macrophage inhibitory cytokine, is a divergent member of the TGF-beta superfamily. Proc Natl Acad Sci USA 94: 11514-11519, 1997.

24. Li PX, Wong J, Ayed A, Ngo D, Brade AM, Arrowsmith C, Austin RC and Klamut HJ: Placental transforming growth factorbeta is a downstream mediator of the growth arrest and apoptotic response of tumor cells to DNA damage and p53 overexpression. J Biol Chem 275: 20127-20135, 2000.

25. Hromas R, Hufford M, Sutton J, Xu D, Li Y and Lu L: PLAB, a novel placental bone morphogenetic protein. Biochim Biophys Acta 1354: 40-44, 1997.

26. Bauskin AR, Brown DA, Junankar S, Rasiah KK, Eggleton S, Hunter M, Liu T, Smith D, Kuffner T, Pankhurst GJ, et al: The propeptide mediates formation of stromal stores of PROMIC-1: Role in determining prostate cancer outcome. Cancer Res 65: 2330-2336, 2005. 
27. Welsh JB, Sapinoso LM, Kern SG, Brown DA, Liu T, Bauskin AR, Ward RL, Hawkins NJ, Quinn DI, Russell PJ, et al: Large-scale delineation of secreted protein biomarkers overexpressed in cancer tissue and serum. Proc Natl Acad Sci USA 100: 3410-3415, 2003

28. Paralkar VM, Vail AL, Grasser WA, Brown TA, Xu H, Vukicevic S, Ke HZ, Qi H, Owen TA and Thompson DD: Cloning and characterization of a novel member of the transforming growth factor-beta/bone morphogenetic protein family. J Biol Chem 273: 13760-13767, 1998.

29. Yin T, Cho SJ and Chen X: RNPC1, an RNA-binding protein and a p53 target, regulates macrophage inhibitory cytokine-1 (MIC-1) expression through mRNA stability. J Biol Chem 288 23680-23686, 2013.

30. Wiklund FE, Bennet AM, Magnusson PK, Eriksson UK, Lindmark F, Wu L, Yaghoutyfam N, Marquis CP, Stattin P, Pedersen NL, et al: Macrophage inhibitory cytokine-1 (MIC-1/ GDF15): A new marker of all-cause mortality. Aging Cell 9: 1057-1064, 2010

31. Staff AC, Trovik J, Eriksson AG, Wik E, Wollert KC, Kempf T and Salvesen HB: Elevated plasma growth differentiation factor-15 correlates with lymph node metastases and poor survival in endometrial cancer. Clin Cancer Res 17: 4825-4833, 2011.

32. Schiegnitz E, Kämmerer PW, Koch FP, Krüger M, Berres M and Al-Nawas B: GDF 15 as an anti-apoptotic, diagnostic and prognostic marker in oral squamous cell carcinoma. Oral Onco 48: 608-614, 2012

33. Tsui KH, Chang YL, Feng TH, Chung LC, Lee TY, Chang PL and Juang $\mathrm{HH}$ : Growth differentiation factor-15 upregulates interleukin- 6 to promote tumorigenesis of prostate carcinoma PC-3 cells. J Mol Endocrinol 49: 153-163, 2012.

34. Grigorian M, Andresen S, Tulchinsky E, Kriajevska M, Carlberg C, Kruse C, Cohn M, Ambartsumian N, Christensen A, Selivanova $\mathrm{G}$, et al: Tumor suppressor $\mathrm{p} 53$ protein is a new target for the metastasis-associated Mts1/S100A4 protein: Functional consequences of their interaction. J Biol Chem 276: 22699-22708, 2001.
35. Tan M, Wang Y, Guan K and Sun Y: PTGF-beta, a type beta transforming growth factor (TGF-beta) superfamily member, is a p53 target gene that inhibits tumor cell growth via TGF-beta signaling pathway. Proc Natl Acad Sci USA 97: 109-114, 2000.

36. Osada M, Park HL, Park MJ, Liu JW, Wu G, Trink B and Sidransky D: A p53-type response element in the GDF15 promoter confers high specificity for $\mathrm{p} 53$ activation. Biochem Biophys Res Commun 354: 913-918, 2007.

37. Baek SJ, Horowitz JM and Eling TE: Molecular cloning and characterization of human nonsteroidal anti-inflammatory drugactivated gene promoter. Basal transcription is mediated by Sp1 and Sp3. J Biol Chem 276: 33384-33392, 2001.

38. Baek SJ, Kim JS, Moore SM, Lee SH, Martinez J and Eling TE: Cyclooxygenase inhibitors induce the expression of the tumor suppressor gene EGR-1, which results in the up-regulation of NAG-1, an antitumorigenic protein. Mol Pharmacol 67: 356-364, 2005.

39. Lee DH, Yang Y, Lee SJ, Kim KY, Koo TH, Shin SM, Song KS, Lee YH, Kim YJ, Lee JJ, et al: Macrophage inhibitory cytokine-1 induces the invasiveness of gastric cancer cells by up-regulating the urokinase-type plasminogen activator system. Cancer Res 63: 4648-4655, 2003.

40. Baek KE, Yoon SR, Kim JT, Kim KS, Kang SH, Yang Y, Lim JS, Choi I, Nam MS, Yoon M, et al: Upregulation and secretion of macrophage inhibitory cytokine-1 (MIC-1) in gastric cancers. Clin Chim Acta 401: 128-133, 2009.

41. Blanco-Calvo M, Tarrío N, Reboredo M, Haz-Conde M, García J, Quindós M, Figueroa A, Antón-Aparicio L, Calvo L and Valladares-Ayerbes M: Circulating levels of GDF15, MMP7 and $\mathrm{miR}-200 \mathrm{c}$ as a poor prognostic signature in gastric cancer. Future Oncol 10: 1187-1202, 2014

42. Tanno T, Lim Y, Wang Q, Chesi M, Bergsagel PL, Matthews G, Johnstone RW, Ghosh N, Borrello I, Huff CA, et al: Growth differentiating factor 15 enhances the tumor-initiating and selfrenewal potential of multiple myeloma cells. Blood 123: 725-733, 2014. 